\title{
CEREBRAL ABSCESS CAUSED BY CLADOPHILAPHORA BANTIANA IN A CHRONIC RENAL FAILURE PATIENT
}

\author{
R. Vidhya Rani, R. Natarajan, J.A. Vasantha Kumar, G. Sekar
}

1. Clinical Microbiologist. Department of Clinical Microbiology, Neuro Foundation, Salem, Tamilnadu.

2. Neuro Surgeon. Department of Clinical Microbiology, Neuro Foundation, Salem, Tamilnadu.

3. General Physician. Department of Clinical Microbiology, Neuro Foundation, Salem, Tamilnadu.

4. Anaesthesiologist. Department of Clinical Microbiology, Neuro Foundation, Salem, Tamilnadu.

\section{CORRESPONDING AUTHOR:}

Dr. R. Vidhya Rani,

Flat A2, Srivijay Anand paradise,

95/9 Gandhi Road,

Salem- 636007.

E-mail: vidhyaranipsg@yahoo.co.in

ABSTRACT: Cladophialophora bantiana is a rare cause of cerebral phaeohyphomycosis in both normal and immunocompromised patients leading to high mortality. We report this rare case report of cerebral abscess caused by these dematiaceous fungi in 47year old male patient, a case of chronic renal failure with end stage renal disease an immunocompromised state along with Hepatitis C infection. Radiological investigations revealed the presence of mature abscess in left parietal cortex. Microcraniotomy with stereotactic aspiration of brain abscess was done. Fungal culture confirmed the etiology as Cladophialophora bantiana. The patient was started on antifungal agents. But the patient could not be successfully treated as he succumbed to his disease.

KEY WORDS: Cladophialophora bantiana, cerebral abscess, immunocompromised

INTRODUCTION: Cerebral phaehyphomycosis caused by Cladophialophora bantiana is a rare fatal disease occurring in both immunocompromised as well as immunocompetent individuals. [1-8] Also known as Xylohypha bantiana it is a dematiaceous fungi ie filamentous fungi with pigmented hyphae due to presence of dihydroxynaphthalene melanin in their cell wall with a remarkable neurotropism causing brain abscess. $[1,3,4]$ The fungus is ubiquitous found in soil, wood and plant matter. ${ }^{[3,6]}$ The organism appears to be acquired through respiratory tract followed by hematogenous dissemination to brain [1,3]. We present a rare case of cerebral abscess in a chronic renal failure patient with end stage renal disease with Hepatitis $\mathrm{C}$ infection which is a immunocompromised condition.

CASE REPORT: A 47year old male known case of chronic renal failure for the past 10 years with chronic kidney disease stage 5 was admitted to our hospital on 17/5/12 for complaints of right sided weakness for past 22 days. He was referred from a peripheral hospital where he was undergoing dialysis regularly for past 5 years. Patient was known to be infected with hepatitis $\mathrm{C}$ since 2 years. Clinical examination of the patient revealed right sided hemiplegia. On admission the patient was afebrile, drowsy with a Glasgow coma score of $11 / 15$. His pulse was $87 /$ minute and blood pressure $180 / 100 \mathrm{~mm} \mathrm{Hg}$. Both pupils were equal and reacting to light. The rest of the physical examination of the patient was normal.

Patient was positive for anti-HCV IgM and seronegative for HIV-1\& HIV-2. Blood investigation showed elevated serum urea and creatinine. His complete blood count, serum 
electrolytes, plasma glucose were normal. 1.5 tesla MRI showed a well defined thick walled nodule suggestive of mature abscess in high parietal cortex. Multislice CT revealed a well defined thin walled irregularly marginated necrotic SOL of size $27 \times 32 \mathrm{~mm}$ with surrounding edema in left area centrum semiovale causing compression of underlying lateral ventricle with marked shift of midline structures to right side [Figure 1]. Minicraniotomy and stereotactic guided aspiration done on 19/5/12.

Direct Gram stain of pus sample revealed plenty of pus cells and pigmented septate hyphae and no bacteria. $10 \%$ potassium hydroxide preparation showed numerous septate hyphae[Figure 2]. Ziehl Neelsen staining was negative for acid fast bacilli. Sample was inoculated into Sabouraud dextrose agar (SDA) and incubated at $37^{\circ} \mathrm{C}$ and at room temperature. After 4 days, the SDA tube incubated at $37^{\circ} \mathrm{C}$ showed moderate growth of colonies that are olive grey with a black reverse [Figure 3]. Lactophenol cotton blue preparation from the fungal colonies revealed pigmented septate hyphae with ellipsoidal to spindle shaped conidia arranged in chains suggestive of Cladophialophora bantiana [Figure 4]. Slide culture showed spindle shaped conidia arranged in long chains confirming the diagnosis [Figure 5]. Fine needle aspiration cytology showed neutrophils, few lymphocytes and filamentous branching fungal hyphae. His urine culture, blood culture for fungus was negative. Patient was started on fluconazole $200 \mathrm{mg}$ twice daily based on Gram staining and Potassium hydroxide report. Patient was also put on antihypertensive and antibiotics. Amphotericin B was not given due to chronic renal failure with raised creatinine level. Though the patient was conscious his conditioned deteriorated and he died on eighth postoperative day.

DISCUSSION: Cladophialophora bantiana is the most commonly isolated causative agent of cerebral phaeohyphomycosis which likely reflects its worldwide distribution. ${ }^{\text {[3] The fungus }}$ existing as saprophytes in soil and decaying vegetation are reported in individuals from rural settings possibly as a result of more prevalent soil exposure. [1,3]. Our patient was from a rural background. The organism appears to be acquired through respiratory tract followed by hematogenous dissemination to brain. Melanin found in cell walls of these fungus act as virulence factor by scavenging free radicals and hypochlorite produced by phagocytic cells that would normally kill most organisms. ${ }^{[1,3,6]}$

Revenkar et al [3] reviewed 101 cases of primary central nervous system phaeohyphomycosis in which Cladophilaphora bantiana accounted for 48 cases and caused cerebral abscess in almost all cases which reflects the strong neurotropic potential of the pathogen.

The lesion is usually located in the frontoparietal lobes and is either well demarcated or poorly circumscribed. [4] Most patients present with symptoms and physical findings compatible with intracerebral mass lesion, focal neurological deficit being the common clinical presentation. $[1,4,3,9]$ Single lesion was present in majority of cases of brain abscess.

The course of disease is generally slow and diagnosis is confirmed by aspiration or surgical resection followed by isolation of fungus. [2, 4] Survival rates were higher when complete resection of lesion was possible in conjunction with systemic antifungal agents. [1,3, 4,] There is as such no standard treatment regime described to treat brain abscess caused by Cladophialophora bantiana although a combination of amphotericin B, flucytosine and azoles have been associated with improved survival rate. [1,10] Previously reported cases of cerebral abscess caused by this fungus were associated with high mortality of about $70 \%$ which may be attributed to difficulty in early diagnosis of this fungal infection, delay in treatment and non 
availability of effective antifungals agents against the organism. [2,3,7] Our patient also could not be successfully treated for this fungal infection whose immunity had been suppressed due to chronic renal failure with end stage renal disease along with Hepatitis $\mathrm{C}$ infection.

\section{REFERENCES:}

1. Bokar SA, Sharma MS, Rajpal G, Jain M, Xess I, Sharma BS . Indian J Med Microbiol $2008 ; 26: 271-4$.

2. Jayakeerthi SR, Dias M, Nagarathna S, Anandh B, Mahadevan A, Chandramuki A. Brain abscess due to cladophialophora bantiana. Indian J Med Microbiol 2004;22:193-5

3. Revankar SG, Sutton DA, Rinaldi MG. Primary cenrtral nervous system phaeohyphomycosis: A review of 101 cases. Clin Infect Dis 2004;38:206-16

4. Hamill RJ. Fungal infections of central nervous system. In Aniassie EJ, McGinnis MR, Pfaller MA. Clinical Mycology second edition (Churchill livingstone, Elsevier, London UK)2010:600-

5. Levin TP. Baty DE, Fekete T, Traunt AL, Suh B. Cladophialophora bantiana brain abscess in a solid-organ transplant recipient: case report and review of literature. J Clin Microbiol 2004:42; 374-8.

6. Alhabib KF, Bryce EA. Xylohypha bantiana multiple brain abscesses in a patient with Systemic lupus erythematosus. Can J Infect Dis2003:14(2);119-120

7. Levin TP, Baty DE, Fekete T, Traunt AL, Suh B. Cladophilalophora bantiana brain abscess in a solid-organ transplant recipient: Case report and review of the literature. J Clin Microbiol 2004; 42: 4374-8

8. Sood P, Dogra V, Thakur A, Mishra B, Mandal A, Sinha S .Brain abscess due to xylohypha bantiana. Scand J Infect Dis 2000; 32:708-9

9. George IA, Mathews MS, Karthik R, John L, Sundar A, Abraham OC, Joseph V. fatal cerebral abscess caused by Cladophialophora bantiana. J Aca Phys India 2008; 56;470472

10. Lyons MK, Blair JE, Leslie KO. Successful treatment with voricanazole of fungal cerebral abscess due to Cladophialaphora bantiana. Clin Neurol neurosurg 2005;107:532-4

[Figure 1] -Multislice CT revealed a well defined thin walled irregularly marginated necrotic SOL of size $27 \times 32 \mathrm{~mm}$ with surrounding edema in left area centrum semiovale.

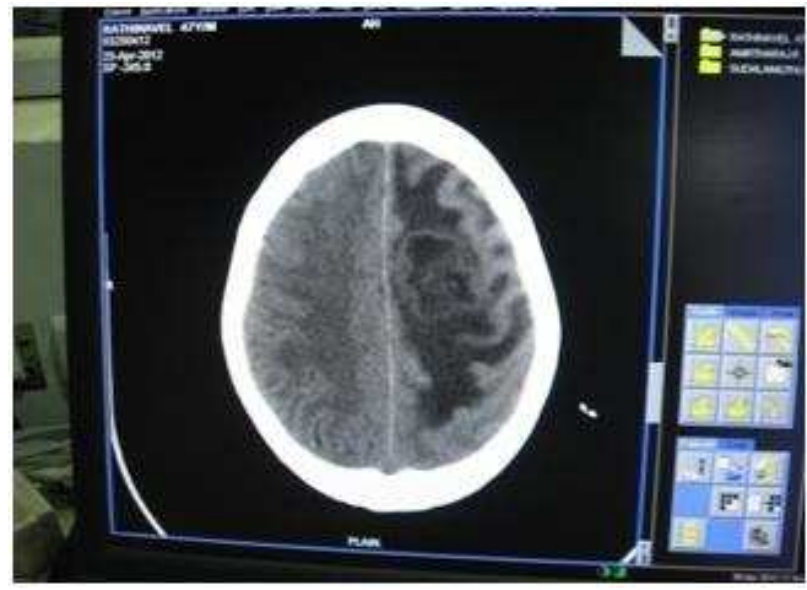


[Figure 2]- 10\% KOH showing septate hyphae

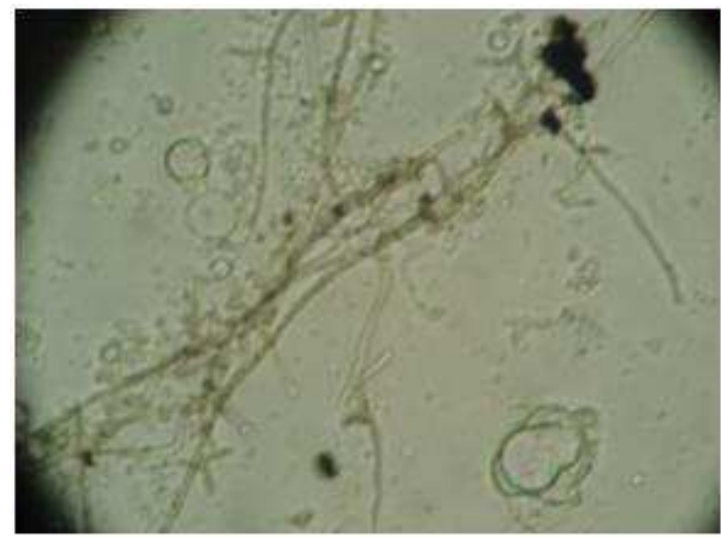

[Figure 3]-: Sabouraud dextrose agar showing olivaceous grey coloured colonies of Cladophialophora bantiana

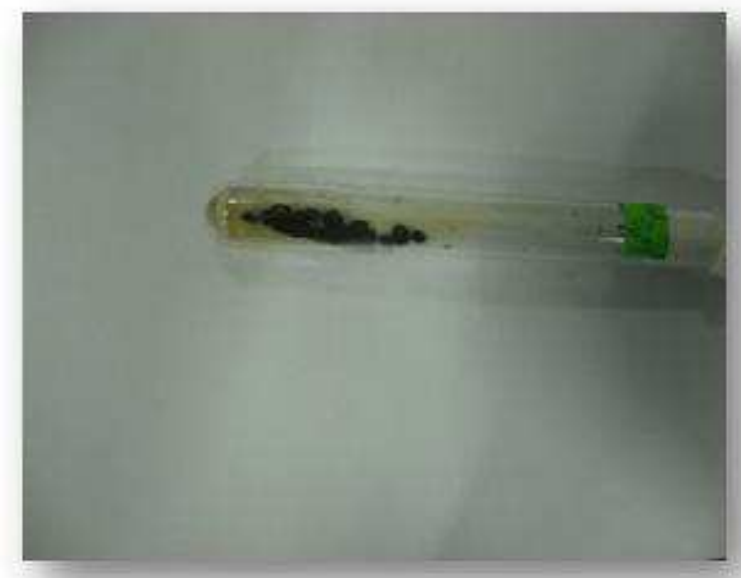

[Figure 4]- Lacto phenol cotton blue mount showing spindle shaped conidia

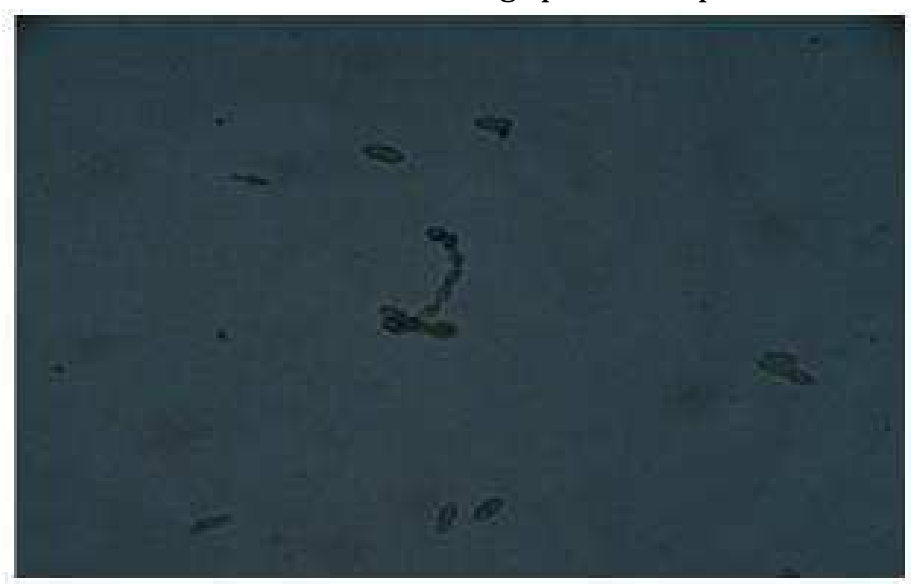


[Figure 5]- Slide culture showing spindle shaped conidia arranged in long chains

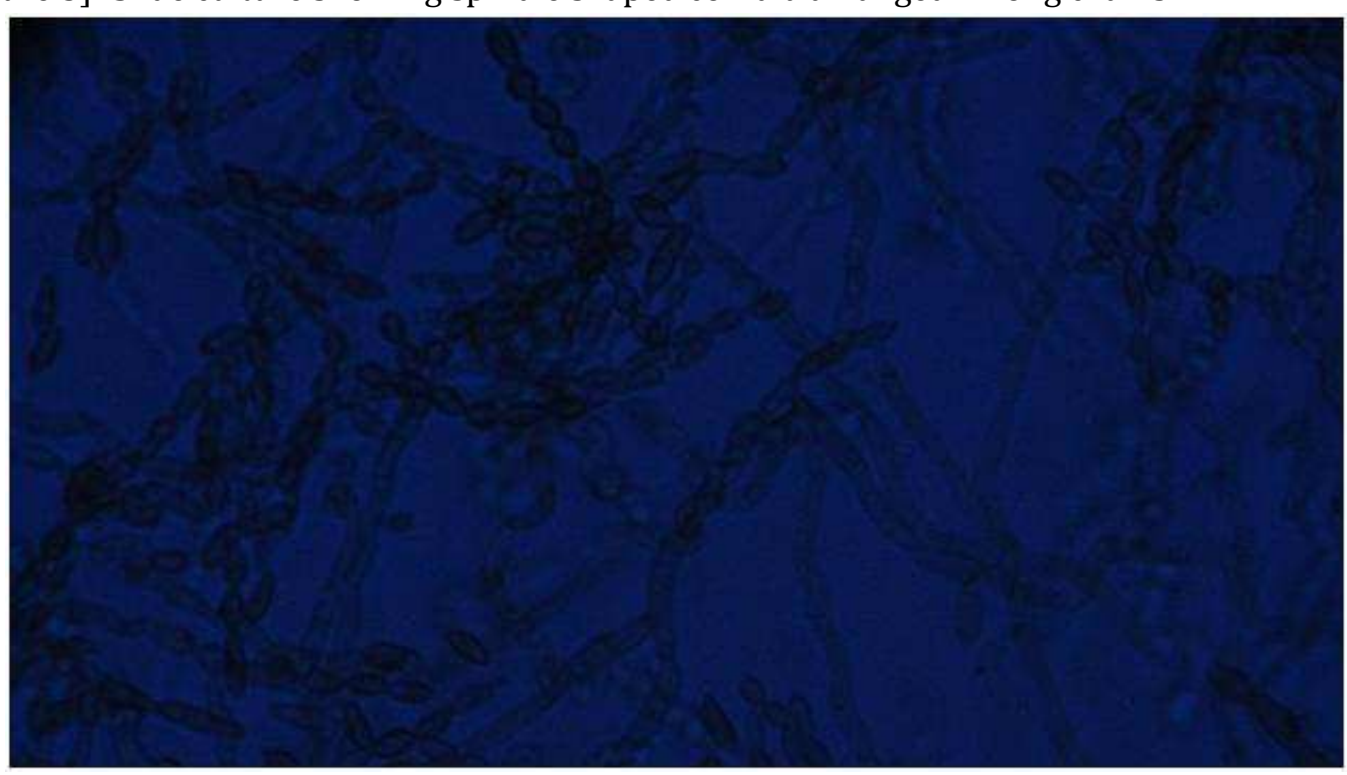

